\title{
Mitigation of radiation myelopathy and reduction of microglial infiltration by Ramipril, ACE inhibitor
}

\author{
Mariano G. Clausi $\mathbb{D}^{1} \cdot$ Alexander M. Stessin ${ }^{1,2} \cdot$ Stella E. Tsirka ${ }^{2} \cdot$ Samuel Ryu ${ }^{1,2}$
}

Received: 19 February 2018 / Revised: 16 April 2018 / Accepted: 22 April 2018 / Published online: 14 June 2018

(c) International Spinal Cord Society 2018

\begin{abstract}
Study design Experimental study.

Objectives To evaluate the efficacy of Angiotensin-converting enzyme inhibitor Ramipril, as a mitigator of radiationinduced spinal cord injury.

Setting Stony Brook University, Stony Brook, NY, USA.

Methods Total of 22 rats were irradiated with single doses of 23.6-33 Gy at the C4-T2 spinal levels. After irradiation, the rats were randomized to the radiation only control group and the Ramipril-treated (radiation + Ramipril) experimental group. Ramipril $1.5 \mathrm{mg} / \mathrm{kg} / \mathrm{day}$ was given in the drinking water starting 1 week after radiation through the study duration.

Results All the rats irradiated with $28.5-33$ Gy became paralyzed at $125 \pm 4$ days, whereas no rats became paralyzed after 23.6 Gy. The time to develop paralysis was delayed to $135 \pm 4$ days in Ramipril-treated group $(P<0.001)$. H\&E and LFB showed microscopic structural restoration and remyelination with Ramipril treatment. VEGF expression was increased in the irradiated spinal cord, and the number of VEGF-positive cells was significantly decreased by Ramipril treatment $(P<0.001)$. Immunohistochemical stain with Iba-1 showed increased microglial infiltration in the irradiated spinal cords. The number of Iba-1-positive microglia was significantly reduced by Ramipril treatment $(P<0.05)$.

Conclusion Ramipril reduced the rate of paralysis even at the paralysis-inducing radiation doses. It also significantly delayed the onset of paralysis. Neuroinflammation and endothelial cell damage may be the key mediators of radiation injury. Ramipril can be readily translatable to clinical application as a mitigatory of radiotherapeutic toxicity.
\end{abstract}

\section{Introduction}

Over $10 \%$ of cancer patients ultimately develop metastatic spinal cord compression. If left untreated, a tumor in the spine can quickly progress to paralyze the patient. Radiotherapy has long been used as an effective treatment for tumors involving both the spinal column and spinal cord. Yet this therapy carries a grave risk of causing myelopathy and irreversible neurological deficits. Because of this consideration, the conventional tolerance

Samuel Ryu

samuel.ryu@stonybrookmedicine.edu

1 Department of Radiation Oncology, Stony Brook University Hospital, Stony Brook, NY, USA

2 Department of Pharmacological Sciences, Stony Brook University Hospital, Stony Brook, NY, USA dose for spinal cord has been accepted as 45-50 Gy in 25 fractions. However, accumulated clinical experience indicates that the spinal cord may tolerate higher radiation doses. Recently the use of cutting edge technology has allowed the introduction of stereotactic radiosurgery to the treatment of spinal tumors, and this new approach has necessitated re-examination of the spinal cord tolerance in the context of radiosurgical and hypofractionated doses. With this platform of radiosurgery in humans, the partial volume tolerance dose has been established at $10 \mathrm{~Gy}$ to $10 \%$ of the ventral part of the spinal cord. This was found to be safe in clinical practice [1] and has been adopted in the RTOG 0631 clinical trial of spine radiosurgery [2].

The mechanism of radiation-induced myelopathy is not well understood. The classic radiobiological models have implicated parenchymal cell loss and vascular changes, yet a number of other mechanisms have been proposed as well. White matter necrosis may be one of the 
A

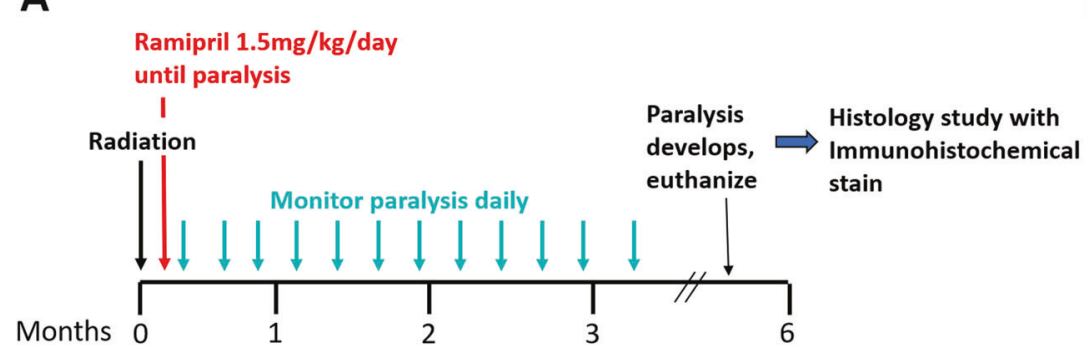

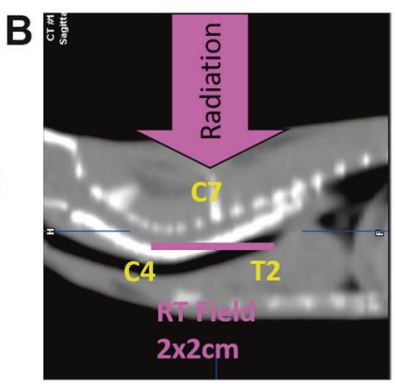

Fig. 1 Schematic representation of the experimental protocol. A A single radiation dose of $23.6,28.5,30$ or $33 \mathrm{~Gy}$ was delivered to a 2 $\mathrm{cm}$ segment of the spinal cord between $\mathrm{C} 4$ and $\mathrm{T} 2$. Ramipril was given in drinking water $(1.5 \mathrm{mg} / \mathrm{kg} /$ day $)$ starting 1 week after irradiation (RT + RAM). B Radiation field position. It was verified with a radiographic film most common late histopathologic changes associated with irreversible neurological deficits [3]. More recent studies suggest a highly complicated pathogenic process involving vascular abnormalities, such as neovascularization, vessel wall thickening, vessel dilation, increased permeability and breakdown of the blood-spinal cord barrier [4]. Radiationinduced myelopathy is generally considered to be irreversible, and at present there are no established treatments available once the toxicity develops. In preclinical studies, one of the most promising approaches to attenuate late radiation injury involves inhibition of the metabolic pathway of the renin-angiotensin system (RAS). The precursor Angiotensin I is converted to Angiotensin II by angiotensin-converting enzyme (ACE). Angiotensin II signals through type 1 and type 2 receptors (AT1 and AT2). By using ACE inhibitors or blocking downstream angiotensin receptors, we and other investigators have shown mitigation of radiation-induced normal tissue toxicity in several organ systems including the optic nerve model, brain and kidney [5-9]. The mechanism of radiation mitigating effect remains to be fully understood, but it may also involve regulation of radiation-induced neuroinflammation [10]. In fact, recent evidence indicates that neuroinflammation by microglial activation may be an important contributing factor in the development of radiation-induced cognitive impairment [11]. In the present study, we hypothesized that treatment with the ACE inhibitor, Ramipril, would mitigate long-term radiation myelopathy, and that post-radiation neuroinflammation within the irradiated spinal cord may play a role. Using a rat model of radiation-induced spinal cord injury, we demonstrate that post-radiation Ramipril treatment results in functional motor recovery as well as microscopic structural restoration of the spinal cord after paralysis-inducing high doses of radiation. The Ramipril treatment also produces a significant reduction in microglial infiltration and vascular endothelial growth factor (VEGF) expression in the irradiated spinal cord tissue.

\section{Methods}

\section{Animal subjects and treatment with Ramipril}

Adult male Fisher 344 rats (Charles River Breeding Lab, Wilmington, MA) weighing between 200 and $240 \mathrm{~g}$ were used. All animal treatments were conducted in accordance with approved protocols reviewed by the institutional animal care committee of Henry Ford Hospital when an author (SR) was there. Rats were anesthetized with intraperitoneal injection (i.p.) of sodium pentobarbital $(50 \mathrm{mg} / \mathrm{kg})$ and immobilized using polystyrene foam. Single radiation doses of $23.6,28.5,30$ or $33 \mathrm{~Gy}$ were delivered using a single collimated dorsoventral beam, centered to a $2-\mathrm{cm}$ segment of the spinal cord between $\mathrm{C} 4$ and $\mathrm{T} 2$. The dose rate from a $6 \mathrm{MV}$ linear accelerator was $2.5 \mathrm{~Gy} / \mathrm{min}$ at $100 \mathrm{~cm}$ source to the surface distance. After irradiation with high doses (30 and $33 \mathrm{~Gy}$ ), total of 22 rats were randomized to the Radiation only group $(n=12)$ and the Ramipril-treated (Radiation + Ramipril) group $(n=10)$. Sham control animals were also anesthetized but did not receive radiation nor Ramipril. Ramipril (Sigma; purity $>98 \%$ ) was administered starting at 1 week after radiation to the spinal cord. Ramipril was given in the drinking water at a concentration of 1.5 $\mathrm{mg} / \mathrm{kg}$ per day which was available at libitum. Ramipril treatment continued until the rats developed paralysis or until they were euthanized. Freshly prepared solution was given every day during the study period. The schema of the experimental design is shown in Fig. 1. Ramipril has highly predictable bioavailability, stability of the drug in water and its ability cross the blood brain barrier, and therefore bloodspinal cord barrier [12].

\section{Monitoring of neurological signs}

The animals were monitored daily by the monitoring personnel of the animal facility who were blinded to the intervention for signs of neurological deficits and paralysis during the planned study duration of 6 months. For each rat, 


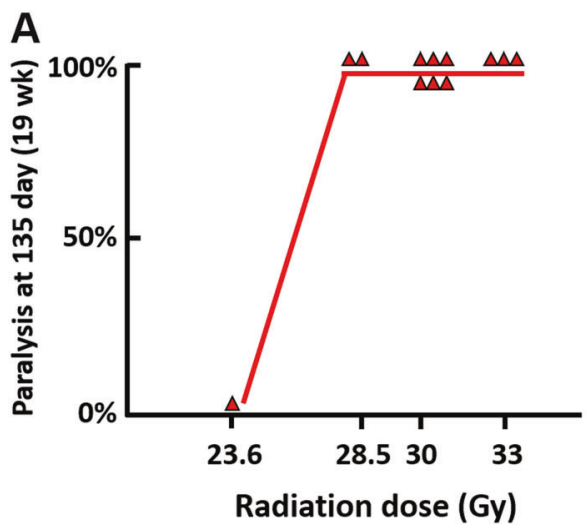

Fig. 2 Radiation-induced paralysis attenuated by Ramipril treatment. A Rate of paralysis at 135 days after radiation to the spinal cord as a function of radiation dose. Each dot represents a single animal $(n=$

the study ended once the rats developed the first sign of paralysis involving one of both fore- and/or hind-limbs or were unable to support the full weight of the body. They were then euthanized according to the IACUC guidelines.

\section{Histology and immunohistochemistry}

After developing paralysis, the rats were anesthetized with an intraperitoneal injection of sodium pentobarbital $(50 \mathrm{mg} /$ $\mathrm{kg}$ ) followed by intracardiac perfusion with PBS and $4 \%$ paraformaldehyde in PBS. The spinal cords were dissected and embedded in paraffin blocks. Each spinal cord was cross sectioned ( $6 \mu \mathrm{m}$ thick) using a microtome and mounted on glass slides. Slides were deparaffinized in xylene and rehydrated through graded alcohol and distilled water. Adjacent serial paraffin sections were stained with hematoxylin and eosin (H\&E) for histopathological assessment and Luxol Fast Blue/ Periodic Acid Schiff to evaluate myelination. To study the neuroinflammation with microglial infiltration and the endothelial and vascular effects, deparaffinized and rehydrated slides were heated in antigen unmasked solution (Vector, Burlingame, CA). Immunofluorescence staining was performed by overnight incubation at $4{ }^{\circ} \mathrm{C}$ with the following antibodies: rabbit polyclonal anti-Iba-1 (1:400; 019-19741, Wako Chemicals, Richmond, VA) and rabbit polyclonal anti-VEGFA (1:100; ab46154, Millipore, Temecula, CA). Secondary antibody against rabbit was incubated for $2 \mathrm{~h}$ at room temperature (Jackson, West Grove, PA). 4,6-Diamidino-2-phenylindole (Sigma, $1 \mu \mathrm{g} / \mathrm{ml}$ ) was used for $15 \mathrm{~min}$ to counterstain nuclei.

\section{Histological analysis and cell quantification}

H\&E sections spanning the region of spinal cord exposed to radiation were evaluated by a reader blinded to specimen identity. Grey and white matter were evaluated by light microscopy for the presence of vacuolation, hemorrhages

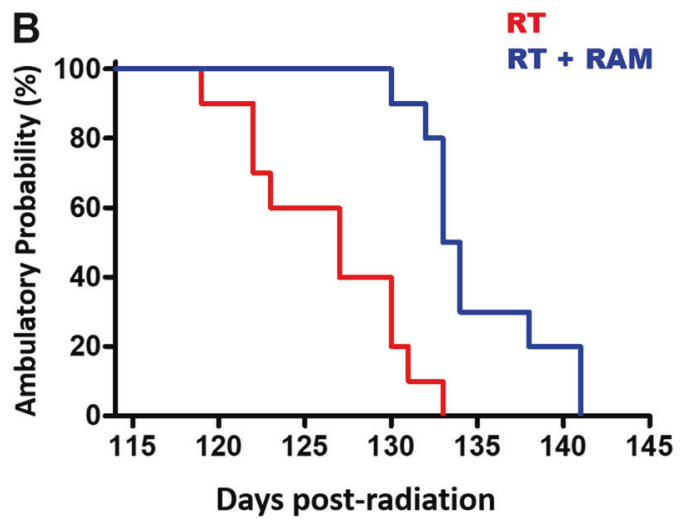

12). B Ambulatory probability curves show time to onset of paralysis after high radiation doses (30 and $33 \mathrm{~Gy}$ ) in RT (red line, $n=12$ ) and $\mathrm{RT}+\mathrm{RAM}$ (blue line, $\mathrm{n}=10$ ). $P<0.001$ by Log-rank test

and necrosis. Histological scores were assigned qualitatively, under low magnification, as mild (score 1) for histological changes observed in less than one third area of the spinal cord; moderate (scores 2-3) for histological changes between one third to one half of the spinal cord; severe (scores 4-5) for histological changes in more than one half of the spinal cord area.

For evaluation of demyelination, microscopic pictures of the spinal cord samples were taken after LFB stain using a Nikon Eclipse E600 Light Microscope equipped with a Nikon Digital Camera DSFi1. The total white matter area was calculated by subtracting the gray matter from the total spinal cord sectional areas using NIH Image J software. The percent demyelinated area was calculated as demyelinated area/total white matter area of spinal cord $\times 100$.

For quantification of microglia stained with Iba-1 and cells expressing VEGFA, the positively stained cells were counted in three sections per animal for a minimum of six animals per group. For each section, representative confocal images of the dorsal funiculus were acquired at $40 \times$ magnification with a Leica TCS SP8X confocal microscope and cell counting was performed by randomly applying counting frames $(200 \times 200 \mu \mathrm{m})$. Cell counting results were converted into cells per $200 \mu^{2}$ using Abercrombie's correction formula [13].

\section{Statistical analysis}

Paralysis data were analyzed by Kaplan-Meier curves with significance determined by log-rank test. The number of demyelinated plaques per section and percentage of demyelinated area were analyzed by Student's t test. VEGF and Iba1 positive cell counting were analyzed by one-way analysis of variance followed by Tukey's post-hoc analysis. All statistical analyses were performed using GraphPad Prism 5 software. Differences were considered statistically significant at a $p$-value of $<0.05$. 
We certify that all applicable institutional and governmental regulations concerning the ethical use of animals were followed during the course of this research.

\section{Results}

\section{Ramipril treatment reduced the rate of paralysis and prolonged the time to develop paralysis after radiation to the spinal cord}

To determine dose response and the $\mathrm{ED}_{100}$ for radiationinduced paralysis, single doses of 23.6, 28.5, 30 or $33 \mathrm{~Gy}$ were delivered to the rat spinal cord. There was a sharp dose response for developing paralysis between $23.6 \mathrm{~Gy}$ and $28.5 \mathrm{~Gy}$ during the 6-month duration of the study. None of the rats showed any signs of motor impairment after a single dose of $23.6 \mathrm{~Gy}$ irradiation. However, after the radiation doses of 28.5, 30 and $33 \mathrm{~Gy}, 100 \%$ of the rats treated with radiation (RT) developed limb paralysis at 135 days postradiation (Fig. 2a). Since previous studies had shown that
Ramipril treatment is an effective mitigator of radiationinduced optic neuropathy when administered early after radiation [9], we started the treatment with Ramipril 1 week after radiation to the spinal cord. Ramipril prolonged the latency to develop paralysis after a paralysis-inducing high radiation doses ( 30 and $33 \mathrm{~Gy}$ ) to the spinal cord. The mean $( \pm \mathrm{SD})$ time to develop paralysis after radiation was $125 \pm$ 4 days in the RT group. In contrast, it was lengthened to $135 \pm 4$ days in RT + RAM group $(P<0.001)$ (Fig. 2b).

\section{Ramipril treatment improved radiation-induced demyelination}

Upon gross examination, the irradiated segment of the spinal cord of rats in the RT group showed a mild brown discoloration on the surface (arrows in Fig. 3a). The diameters of the spinal cords were not different among the experimental groups. Histology of H\&E stained sections revealed a bubbly vacuolation in the grey and white matter of the spinal cord in the RT group (Fig. 3a). High magnification images showed hyalinization with areas of

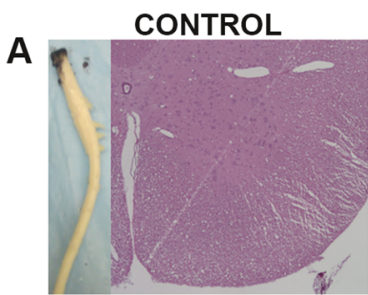

B
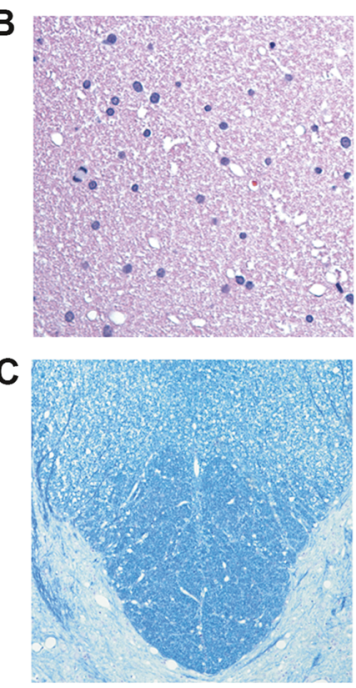
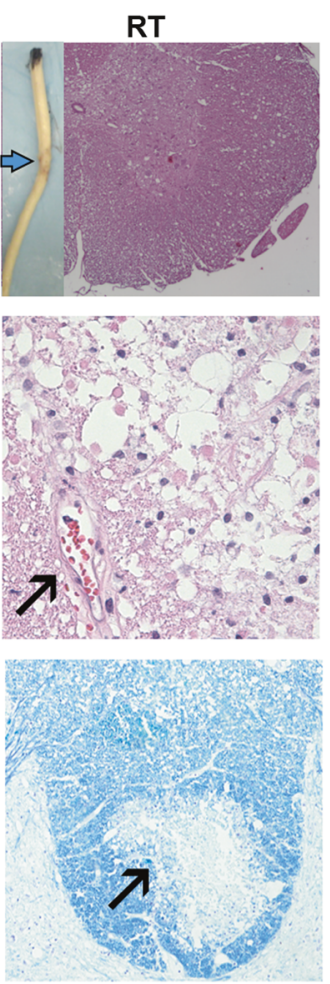

Fig. 3 Ramipril treatment improved radiation-induced demyelination. The animals received paralysis-inducing high radiation doses (30 and $33 \mathrm{~Gy}$ ) and were sacrificed after paralysis. A Representative H\&E stained sections from control (no radiation), RT and RT + RAM, 4x magnification. Insets show pictures of the gross spinal cords. Arrows indicate the region exposed to radiation in RT and RT + RAM animals. B H\&E stains 40× magnification show large vacuoles and swollen axons in RT animals. Arrow point a degenerated and
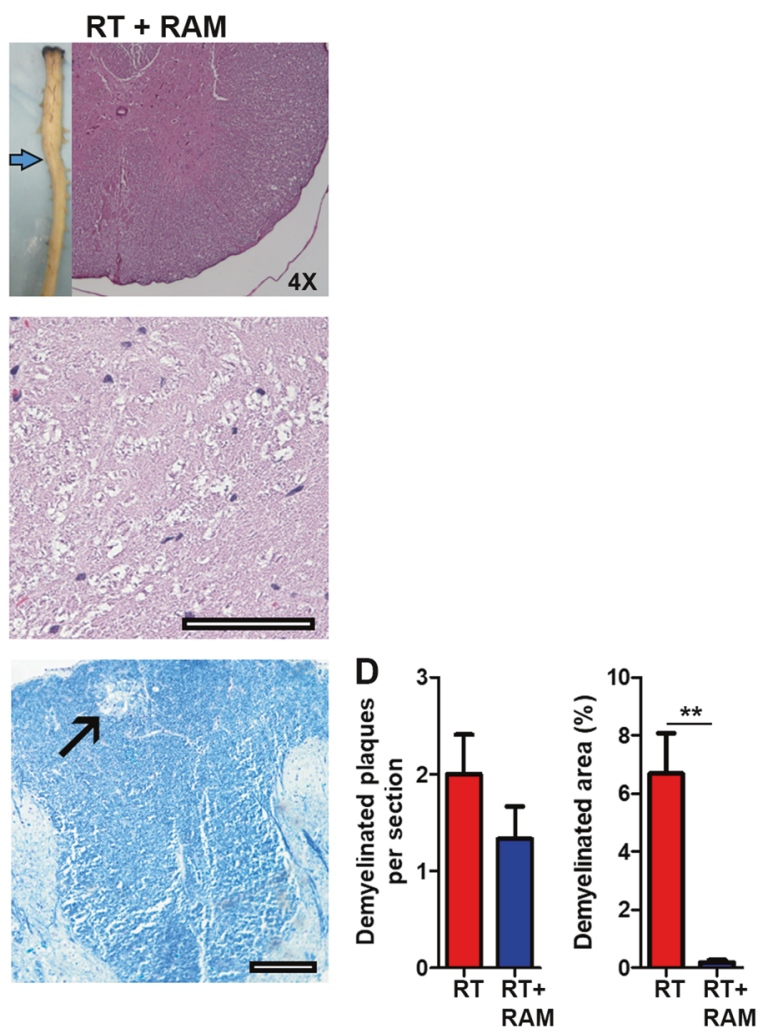

thrombosed blood vessel. Scale bar $=100 \mu \mathrm{m}$. C"?>c LFB stain. Intact myelin in control (no radiation). Arrows indicate areas of demyelination in sections from RT and RT + RAM animals. Scale bar $=200 \mu \mathrm{m}$. D Number of demyelinated plaques and the total area of demyelination per section in RT and RT + RAM animals. Data represent average \pm SEM, $n=9$ per group, $* * P<0.01$ by Student's $t$ test 

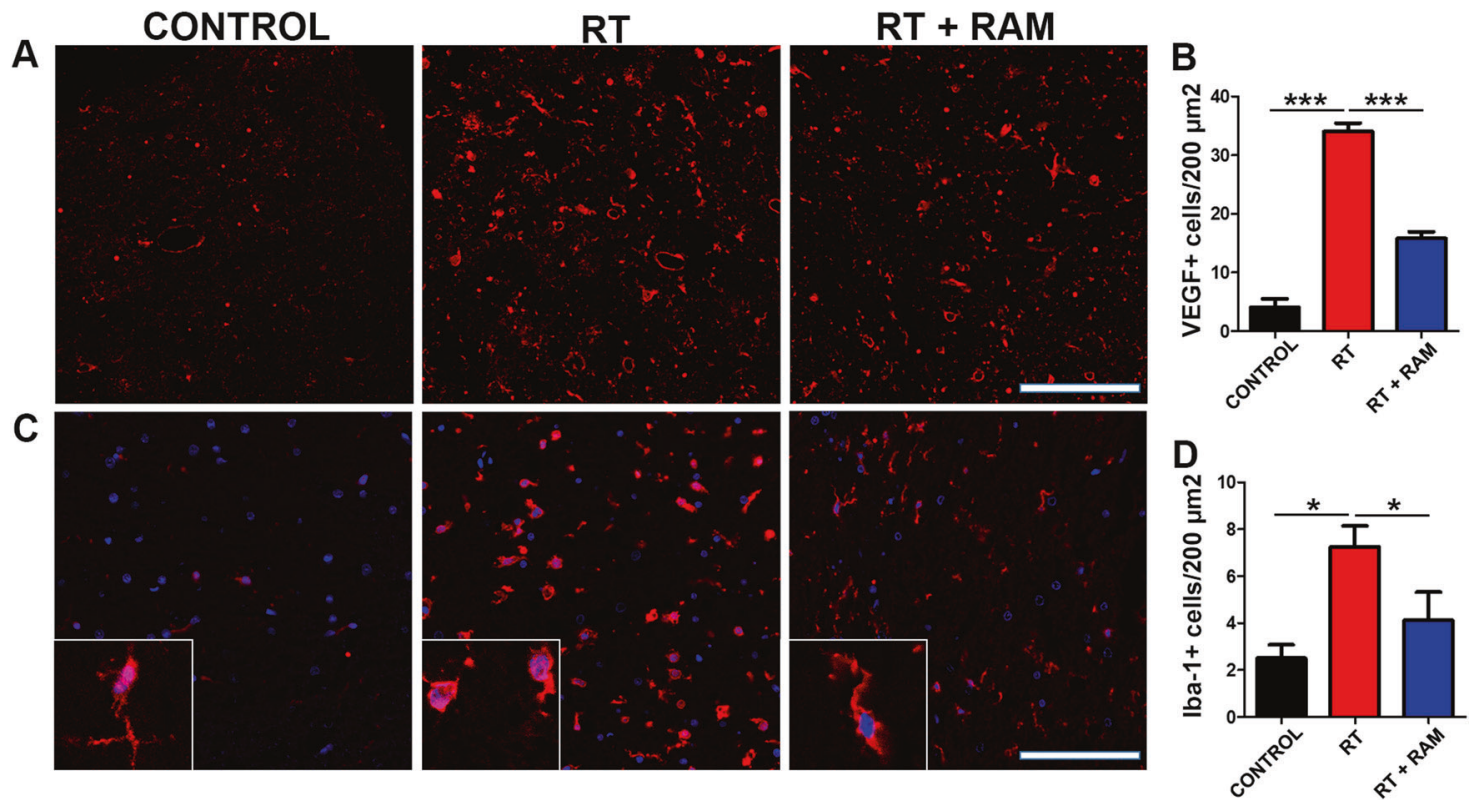

Fig. 4 Ramipril treatment attenuated radiation-induced VEGF expression and Iba-1 expression. The animals received paralysisinducing high radiation doses ( 30 and $33 \mathrm{~Gy}$ ) and were sacrificed after paralysis. A and B Photomicrographs of VEGF stain and quantification of VEGF positive cells in the dorsal column. $\mathbf{C}$ and $\mathbf{D}$

hemorrhage and extensive necrosis in the tissue of irradiated animals. These necrotic areas were associated with prominent vacuolization and axonal swelling. Thrombosed blood vessels were also identified (RT, Fig. 3b). Hemorrhage was present in the grey and white matter, but necrosis was observed only in the white matter. In the Ramipril-treated group, the tissue architecture was better preserved microscopically, and there was less vacuolization (RT + RAM, Figs. 3a, b). The hemorrhage and necrosis were less frequent in both the white and grey matter of Ramipril-treated spinal cords (RT + RAM, Fig. 3b).

LFB stains showed restoration of white matter structural integrity in the Ramipril-treated spinal cord. In irradiated rats an average of two demyelinated plaques per section were identified in the lateral and dorsal column. Ramipril treatment showed a trend to decrease the mean $( \pm \mathrm{SE})$ number of demyelinated plaques per section by $0.7 \pm 0.6$ (95\% CI $0-1.4$ plaques per section) $(P>0.05)$. On the other hand, the mean $( \pm \mathrm{SE})$ area of demyelination was $6.7 \pm 1.4 \%$ of the white matter per cross-section of the spinal cord in the RT group, whereas the demyelinated area was $0.2 \pm 0.1 \%$ of the white matter in the RT + RAM group $(P<0.01)$ (Fig. 3c, d) with a mean between-group difference of $6.5 \%$ (95\% CI $4.5 \%-8.5 \%)$.

\section{Ramipril reduced microglial infiltration and VEGF expression after radiation to the spinal cord}

We found that VEGFA expression was increased throughout the cross sections of the irradiated spinal cord,
Photomicrographs of Iba-1 immunostaining and quantification of Iba-1 positive cells in the dorsal column. Scale bar $=100$ um. Insets in (c) show higher magnification image with microglia $(63 \times)$. Data in $(\mathbf{B})$ and (D) represent average \pm SEM, $n=9$ per group, $* P<0.05$; $* * * P<$ 0.001 by ANOVA followed by Tukey's post hoc test

particularly in the dorsal column. There were also dilated capillaries with endothelium positively stained with antiVEGF. The mean $( \pm$ SE) number of VEGF positive cells was $4 \pm 1$ cells $/ 200 \mu^{2}$ in un-irradiated naïve controls. There was an 8-fold increase in the number of VEGF positive cells to $34 \pm 2$ cells $/ 200 \mu^{2}$ in the RT group $(P<0.001)$ with a mean between-group difference of 29 cells $/ 200 \mu^{2}$ (95\% CI 24-35 cells/200 $\mu^{2}{ }^{2}$ ). In contrast, Ramipril reduced the number of VEGF positive cells to $16 \pm 1$ cells $/ 200 \mu^{2}$ in the $\mathrm{RT}+\mathrm{RAM}$ group $(P<0.001)$ (Fig. 4a, b). The mean difference between RT and RT + RAM was 17 cells $/ 200 \mu \mathrm{m}^{2}$ (95\% CI 12-23 cells/ $200 \mu^{2}$ ).

We evaluated infiltration of microglia/macrophages with Iba-1 staining in the dorsal column. The mean $( \pm \mathrm{SE})$ number of Iba- 1 positive cells was $2 \pm 1$ cells $/ 200 \mu \mathrm{m}^{2}$ in the un-irradiated naïve controls. There was a 3 -fold increase in the number of Iba- 1 positive cells to $7 \pm 1$ cells $/ 200 \mu \mathrm{m}^{2}$ in the RT group $(P<0.05)$ with a mean between-group difference of 5 cells $/ 200 \mu \mathrm{m}^{2}\left(95 \%\right.$ CI $1-9$ cells $\left./ 200 \mu^{2}\right)$. Ramipril treatment significantly reduced the number of Iba1 positive cells to $4 \pm 1$ cells $/ 200 \mu^{2}$ in the RT + RAM group $(P<0.05)$ (Fig. 4c, d). The mean difference between $\mathrm{RT}$ and RT + RAM was 3 cells $/ 200 \mu \mathrm{m}^{2}$ (95\% CI 1-7 cells/ $200 \mu^{2}$ ). Of note, the microscopic morphology of the microglia/macrophages from the RT group displayed an amoeboid shape suggesting an activated phenotype. In contrast, Iba-1 positive cells in the RT + RAM group had a more ramified shape suggesting the less activated 
phenotype similar to those of the unirradiated control group (Insets in Fig. 4c).

\section{Discussion}

To our knowledge this is the first study to demonstrate the mitigating effects of Ramipril in significantly reducing the paralysis rate and delaying the onset of paralysis after irradiation of the spinal cord with paralysis-inducing (ED100) dose. This mitigating effect was well correlated with inhibition of microglial infiltration and VEGF expression.

The dose response curve for radiation-induced paralysis has been well established in different animal models by either partial or full diameter irradiation of the spinal cord $[14,15]$. Consistently with these results, our study revealed steep slope in the dose response curve for paralysis between $23.6 \mathrm{~Gy}$ and $28.5 \mathrm{~Gy}, 4$ months after irradiation of $2 \mathrm{~cm}$ length of the full-diameter cervicothoracic spinal cord. Based on this finding, we chose the paralysis-inducing doses of 30 and $33 \mathrm{~Gy}$ to evaluate the effect of Ramipril in mitigating radiation myelopathy with paralysis as the clinical endpoint.

Multiple studies have demonstrated the mitigating effects of Ramipril for radiation injury to various organs including optic chiasm, hippocampus and whole brain, as well as kidney and skin. Of note, the mitigating effects were seen with different fractionation schedules mimicking a variety of clinical scenarios [7, 9, 16, 17]. Studies performed by Robbins et al. [18] and Moulder et al. [7] showed that hemodynamic changes in the kidneys after radiation could be normalized by ACE inhibitors, suggesting a role of Ang II in the pathogenesis of radiation damage to the kidney. ACE inhibitor treatment also protects the lungs from radiation-induced pneumonitis, fibrosis and pulmonary endothelial damage [16]. In the central nervous system, AT1 receptor antagonist L-158,809 and ACE inhibitor, Ramipril treatment ameliorated deficits in perirhinal cortexdependent cognitive function after whole brain radiation to rats [5]. In a model of radiation-induced optic neuropathy, Ramipril treatment restored the gross and microscopic structural integrity of myelination of the optic nerves. It also resulted in functional improvement as assessed with improvement of the visual evoked potentials [8, 9]. Various mechanisms have been studied in the RAS metabolic pathway, but the exact mechanism of Ramipril mitigation is not clearly understood. Nevertheless, a recent study demonstrated cognitive improvement with complete elimination of microglia by blocking colony-stimulating factor-1 receptor [11]. Consistently with this finding, in our study the mitigating effect of Ramipril for radiation spinal cord damage was correlated with decreased microglia infiltration.
Taken together these results strongly suggest a role for neuroinflammation in the pathogenesis of spinal cord radiation damage. Importantly, the results raise further questions about the temporal relationship of microglial activation during the dynamic process of radiation damage. Further studies are needed to answer the questions of when microglial infiltration/activation begins, where the cells come from (i.e., resident microglia vs. infiltrating macrophages), and the precise role that neuroinflammation plays in mediating radiation-induced paralysis.

Several lines of evidence suggest potential mechanisms for Ramipril's ability to mitigate neuroinflammation. Direct infusion of Ang II to the brain increases adhesion of leukocytes to pial venules, expression of ICAM-1, VCAM and E- and P-selectin adhesion molecules and blood-brainbarrier disruption [19, 20]. In experimental models of atherosclerosis and hypertension, Ang II enhanced the expression of chemokines, MCP- 1 and MIP- $1 \alpha$ resulting in monocyte/macrophage infiltration, and it was ameliorated by ACE inhibitors and AT1 antagonists [21]. On the other hand, Ang II blockade has been shown to suppress microglia activation after LPS stimulation [22]. Ang II stimulates these cellular responses by inducing the expression and DNA-binding capacity of the transcription factor nuclear factor- $\kappa \mathrm{B}(\mathrm{NF}-\kappa \mathrm{B})$ which controls many genes associated with inflammation [23].

It has been shown that radiation causes endothelial damage and decrease in vascular density with subsequent hypoxia, leading to VEGF upregulation [24]. VEGF is a potent endothelial mitogen and permeability factor that induces fenestrations in the endothelium of small venules and capillaries [25, 26] and the expression of adhesion molecules like ICAM-1 [27] further compromising the integrity of the blood-spinal cord barrier. Ang II induces VEGF mRNA production in vascular smooth muscle cells and heart endothelial cells that could be prevented by AT1 blockade [28]. In experimental models of diabetic retinopathy, ACE inhibitors and AT1 antagonists prevent the overexpression of VEGF and hyperpermeability as well as retinal neovascularization [29]. Upregulation of VEGF could be detected at 15 weeks after radiation to the rat spinal cord and precedes the appearance of demyelination and necrosis in the white matter at 18-20 weeks after radiation [24]. Our study demonstrated that Ramipril significantly reduced the VEGF expression, and this was also correlated with the improved outcome of paralysis. The results suggest that Ang II may mediate radiation-induced vascular damage and may also have contributed to the migration of the inflammatory cells to the injured area of the spinal cord through VEGF-induced changes in capillary permeability.

This study is limited by the use of the high paralysisinducing radiation doses which are far above the radiation 
doses used for patients in the clinic. In extrapolating the experimental data for clinical application one must be further cautioned to take into account factors such as patient comorbidities and interactions with concomitant systemic therapies. Nonetheless, Ramipril is a well-tolerated, FDA approved drug that crosses the blood brain barrier [12] and therefore also crosses blood-spinal cord barrier. In conclusion, our study supports the translational potential for using Ramipril as a mitigator of radiation-induced normal tissue toxicity in patients.

Acknowledgements The authors acknowledge that the animal irradiation was performed at Henry Ford Hospital (SR before moving to Stony Brook University), thank Andrew Koloszvary, Stephen Brown, Karen Lapanowski for technical help.

Funding This work was supported by internal funds from Stony Brook University Hospital.

Author contributions MGC was responsible for the methodology, project administration, software, visualization, writing-original draft and writing-review. AMS was responsible for the formal analysis, investigation, methodology, validation, visualization, writing-original draft and writing-review. SET was responsible for supervision, data interpretation, validation, visualization, writing-original draft and writing-review and editing. SR was responsible for the conceptualization, formal analysis, funding acquisition, investigation, methodology, supervision, validation, visualization, writing-original draft and writing-review and editing.

\section{Compliance with ethical standards}

Conflict of interest The authors declare that they have no conflict of interest.

\section{References}

1. Ryu S, Jin JY, Jin R, Rock J, Ajlouni M, Movsas B, et al. Partial volume tolerance of the spinal cord and complications of singledose radiosurgery. Cancer. 2007;109:628-36.

2. Ryu S, Pugh SL, Gerszten PC, Yin FF, Timmerman RD, Hitchcock YJ, et al. RTOG 0631 phase $2 / 3$ study of image guided stereotactic radiosurgery for localized (1-3) spine metastases: phase 2 results. Pract Radiat Oncol. 2014;4:76-81.

3. Schultheiss TE, Stephens LC. The pathogenesis of radiation myelopathy: widening the circle. Int J Radiat Oncol Biol Phys. 1992;23:1089-91.

4. Wong CS, Fehlings MG, Sahgal A. Pathobiology of radiation myelopathy and strategies to mitigate injury. Spinal Cord. 2015;53:574-80.

5. Conner KR, Payne VS, Forbes ME, Robbins ME, Riddle DR. Effects of the AT1 receptor antagonist L-158,809 on microglia and neurogenesis after fractionated whole-brain irradiation. Radiat Res. 2010;173:49-61.

6. Lee TC, Greene-Schloesser D, Payne V, Diz DI, Hsu FC, Kooshki $\mathrm{M}$, et al. Chronic administration of the angiotensin-converting enzyme inhibitor, ramipril, prevents fractionated whole-brain irradiation-induced perirhinal cortex-dependent cognitive impairment. Radiat Res. 2012;178:46-56.
7. Moulder JE, Cohen EP, Fish BL. Mitigation of experimental radiation nephropathy by renin-equivalent doses of angiotensin converting enzyme inhibitors. Int J Radiat Biol. 2014;90:762-8.

8. Kim JH, Brown SL, Kolozsvary A, Jenrow KA, Ryu S, Rosenblum ML, et al. Modification of radiation injury by ramipril, inhibitor of angiotensin-converting enzyme, on optic neuropathy in the rat. Radiat Res. 2004;161:137-42.

9. Ryu S, Kolozsvary A, Jenrow KA, Brown SL, Kim JH. Mitigation of radiation-induced optic neuropathy in rats by ACE inhibitor ramipril: importance of ramipril dose and treatment time. J Neurooncol. 2007;82:119-24.

10. Robbins ME, Zhao W, Garcia-Espinosa MA, Diz DI. Reninangiotensin system blockers and modulation of radiation-induced brain injury. Curr Drug Targets. 2010;11:1413-22.

11. Acharya MM, Green KN, Allen BD, Najafi AR, Syage A, Minasyan $\mathrm{H}$, et al. Elimination of microglia improves cognitive function following cranial irradiation. Sci Rep. 2016;6:31545.

12. Nordstrom M, Abrahamsson T, Ervik M, Forshult E, Regardh CG. Central nervous and systemic kinetics of ramipril and ramiprilat in the conscious dog. J Pharmacol Exp Ther. 1993;266:147-52.

13. Abercrombie M. Estimation of nuclear population from microtome sections. Anat Rec. 1946;94:239-47.

14. Bijl HP, van Luijk P, Coppes RP, Schippers JM, Konings AW, van der Kogel AJ. Dose-volume effects in the rat cervical spinal cord after proton irradiation. Int $\mathrm{J}$ Radiat Oncol Biol Phys. 2002;52:205-11.

15. Medin PM, Foster RD, van der Kogel AJ, Sayre JW, McBride WH, Solberg TD. Spinal cord tolerance to single-fraction partialvolume irradiation: a swine model. Int J Radiat Oncol Biol Phys. 2011;79:226-32.

16. Molteni A, Moulder JE, Cohen EF, Ward WF, Fish BL, Taylor $\mathrm{JM}$, et al. Control of radiation-induced pneumopathy and lung fibrosis by angiotensin-converting enzyme inhibitors and an angiotensin II type 1 receptor blocker. Int $\mathrm{J}$ Radiat Biol. 2000;76:523-32.

17. Kohl RR, Kolozsvary A, Brown SL, Zhu G, Kim JH. Differential radiation effect in tumor and normal tissue after treatment with ramipril, an angiotensin-converting enzyme inhibitor. Radiat Res. 2007; $168: 440-5$

18. Robbins ME, Hopewell JW. Physiological factors effecting renal radiation tolerance: a guide to the treatment of late effects. Br J Cancer Suppl. 1986;7:265-7.

19. Alvarez A, Cerda-Nicolas M, Naim Abu Nabah Y, Mata M, Issekutz AC, Panes J, et al. Direct evidence of leukocyte adhesion in arterioles by angiotensin II. Blood. 2004;104:402-8.

20. Piqueras L, Kubes P, Alvarez A, O'Connor E, Issekutz AC, Esplugues JV, et al. Angiotensin II induces leukocyte-endothelial cell interactions in vivo via $\mathrm{AT}(1)$ and $\mathrm{AT}(2)$ receptor-mediated $\mathrm{P}$ selectin upregulation. Circulation. 2000;102:2118-23.

21. Rahman ST, Lauten WB, Khan QA, Navalkar S, Parthasarathy S, Khan BV. Effects of eprosartan versus hydrochlorothiazide on markers of vascular oxidation and inflammation and blood pressure (renin-angiotensin system antagonists, oxidation, and inflammation). Am J Cardiol. 2002;89:686-90.

22. Benicky J, Sanchez-Lemus E, Honda M, Pang T, Orecna M, Wang J, et al. Angiotensin II AT1 receptor blockade ameliorates brain inflammation. Neuropsychopharmacology. 2011;36:857-70.

23. Ruiz-Ortega M, Lorenzo O, Ruperez M, Suzuki Y, Egido J. Angiotensin II activates nuclear transcription factor-kappaB in aorta of normal rats and in vascular smooth muscle cells of AT1 knockout mice. Nephrol Dial Transplant. 2001;16:27-33.

24. Nordal RA, Nagy A, Pintilie M, Wong CS. Hypoxia and hypoxiainducible factor-1 target genes in central nervous system radiation injury: a role for vascular endothelial growth factor. Clin Cancer Res. 2004;10:3342-53. 
25. Leung DW, Cachianes G, Kuang WJ, Goeddel DV, Ferrara N. Vascular endothelial growth factor is a secreted angiogenic mitogen. Science. 1989;246:1306-9.

26. Dvorak HF, Brown LF, Detmar M, Dvorak AM. Vascular permeability factor/vascular endothelial growth factor, microvascular hyperpermeability, and angiogenesis. Am J Pathol. 1995;146: 1029-39.

27. Radisavljevic Z, Avraham H, Avraham S. Vascular endothelial growth factor up-regulates ICAM-1 expression via the phosphatidylinositol 3 OH-kinase/AKT/Nitric oxide pathway and modulates migration of brain microvascular endothelial cells. J Biol Chem. 2000;275:20770-4.

28. Chua CC, Hamdy RC, Chua BH. Upregulation of vascular endothelial growth factor by angiotensin II in rat heart endothelial cells. Biochim Biophys Acta. 1998;1401:187-94.

29. Wilkinson-Berka JL, Kelly DJ, Gilbert RE. The interaction between the renin-angiotensin system and vascular endothelial growth factor in the pathogenesis of retinal neovascularization in diabetes. J Vasc Res. 2001;38:527-35. 